GENDER DIFFERENCES IN LONELINESS

Running head: GENDER DIFFERENCES IN LONELINESS

Gender Differences in Loneliness Across the Lifespan:

A Meta-Analysis 


\title{
GENDER DIFFERENCES IN LONELINESS
}

\begin{abstract}
Different theoretical contentions on gender differences in loneliness have been proposed, often including the emergence of gender differences in particular developmental periods. However, empirical results have been inconsistent and a lifespan perspective examining those different developmental phases has been missing. The present meta-analysis synthesizes the available evidence on gender differences in loneliness across the lifespan. We analyzed 747 effect sizes from 634 studies published between 1978 and 2016, covering a total of 398,338 individuals (45.61\% men). To account for dependencies among effect sizes, threelevel meta-analyses were conducted. Results showed a close-to-zero overall effect ( $g=0.07$ ). Most of the examined moderators were non-significant, except for age, the scope of the sampling area, and year of publication. Most importantly, all effects were small, suggesting that across the lifespan mean levels of loneliness are similar for men and women. As a result, none of the genders should be targeted more than the other when designing prevention and intervention projects to alleviate loneliness.
\end{abstract}

Keywords: loneliness, gender differences, lifespan, meta-analysis 


\section{GENDER DIFFERENCES IN LONELINESS}

\section{Gender Differences in Loneliness Across the Lifespan: A Meta-Analysis}

Loneliness is defined as the unpleasant feeling that occurs when people perceive their network of social relationships to be deficient in quantitative or qualitative ways (Perlman \& Peplau, 1981). According to the evolutionary theory of loneliness (J. T. Cacioppo et al., 2015), the social pain of loneliness serves as a warning system that (1) signals to people that something is missing in their social relationships and (2) motivates them to reconnect to significant others. However, for some individuals, this reconnection may fail, detrimentally affecting their mental and physical well-being. For example, research has shown that lonely people have more psychological problems, such as depression and anxiety, more physical health problems, such as sleep problems and cardiovascular incidents, become ill more quickly, and pass away at an earlier age (see for reviews Ernst \& Cacioppo, 1999; Goossens et al., 2015; Hawkley \& Capitanio, 2015; Holt-Lunstad, Smith, Baker, Harris, \& Stephenson, 2015). These detrimental effects have mainly been studied in adults, but the poor health effects of loneliness have been found in children and adolescents as well (Doane \& Thurston, 2014; Harris, Qualter, \& Robinson, 2013; Heinrich \& Gullone, 2006; Qualter et al., 2013). Hence, it is important to examine loneliness across the lifespan.

The mounting evidence for direct links to poor health and well-being that has emerged over the last decade has led both researchers and policy makers to pay increasing attention to loneliness. A frequently asked question is whether gender represents a vulnerability factor for loneliness. However, results on this issue have been largely inconsistent and no consensus has been reached. With the focus on ensuring that effective interventions are delivered to the right people, knowing whether males or females are more vulnerable to loneliness is important. Knowing whether and when those gender differences emerge and whether they remain stable over the life course is also essential. Hence, our main 


\section{GENDER DIFFERENCES IN LONELINESS}

question in this meta-analysis is whether there are gender differences in loneliness, and whether that pattern is the same across the course of human life.

\section{Gender Differences in Loneliness}

Some researchers have argued that boys will be lonelier than girls from adolescence onwards (Koenig \& Abrams, 1999). That hypothesis has been explained by arguing that although both male and female adolescents spend less time with their family than children, boys show a steeper decline in family time than girls. Moreover, it has been hypothesized that for girls, time spent with family is replaced with time spent with peers, whereas for boys the reduction in time spent with family leads to more time spent alone - and thus, potentially leads to more loneliness among boys. While it is the case that higher levels of aloneness (i.e., the objective state of being alone) do not necessarily lead to higher levels of loneliness (Larson, 1990), it is possible that during adolescence, at a time of self-discovery and identity development, which develops in reference to peers (Csikszentmihalyi \& Larson, 1984), spending more time alone worsens feelings of disconnection from others and increases the risk of loneliness. Both male and female adolescents are at increased risk of loneliness compared to other age groups because they are experiencing social changes (Qualter et al., 2015). However, boys may be at particular risk because they are increasing the time they spend alone, isolating themselves from both the peer group and the family.

Other researchers have hypothesized that gender differences in loneliness emerge in adolescence, but believe that females will become lonelier than males. This hypothesis is derived from theoretical models of internalizing problems, and is based on the assumption that loneliness can be categorized as an internalizing problem (Creemers, Scholte, Engels, Prinstein, \& Wiers, 2012; Romero \& Epkins, 2008; Vanhalst et al., 2012). The sexual selection evolutionary theory has been used to explain the common finding that women are more at risk for adolescent-onset internalizing problems (Martel, 2013). That theory suggests 


\section{GENDER DIFFERENCES IN LONELINESS}

that adolescence, and in particular puberty, is a critical period for females, because they become more sensitive to interpersonal aspects of the social environment. In addition, the theory states that females have more negative emotionality and more effortful control than males, with both characteristics being linked to the development of internalizing problems.

It has been hypothesized that also during the transitional period of old age, women will be lonelier than men. For instance, in a study exploring gender differences in psychological well-being in old age (Pinquart \& Sörensen, 2001), it was argued that women are more vulnerable to loneliness because they tend to live longer and are, therefore, more likely to be widowed, to struggle with functional limitations (e.g., restricted mobility), and to require more health care.

\section{Moderation of Gender Differences in Loneliness}

The existence and size of gender differences in loneliness may depend on several factors.

Age. As detailed before, the age range or developmental period in which a study is conducted could influence whether gender differences are found. More specifically, gender differences have been hypothesized to emerge during the transition periods of adolescence and old age. However, loneliness research with a lifespan focus is rare, and it is yet unclear whether gender differences that are thought to emerge in adolescence remain stable or become smaller or larger across adulthood.

Loneliness types. Gender differences may also vary according to the type of loneliness that is examined. Three types of loneliness have been distinguished in the literature, integrating different previous categorizations of types of loneliness. These types are referred to as intimate, relational, and collective loneliness (S. Cacioppo, Grippo, London, Goossens, \& Cacioppo, 2015). Intimate - or emotional - loneliness is the feeling of lacking a close, intimate attachment to another person. That perceived absence of a significant other may refer to different relationships across the lifespan, including a parent, best friend, or a romantic 


\section{GENDER DIFFERENCES IN LONELINESS}

partner. Relational - or social - loneliness is the feeling of lacking a network of social relationships, and may refer to different networks, including a family, a group of friends, or classroom peers. Collective loneliness refers to experienced discrepancies in one's valued social identities and connections with similar others. Those similar others are not necessarily known and constitute broader groups, such as one's school, neighborhood, or cultural group. Because women orient more toward dyadic, intimate attachments (Baumeister \& Sommer, 1997; Gardner \& Gabriel, 2004), they might experience less intimate loneliness than men (Hoza, Bukowski, \& Beery, 2000). However, one could also argue that precisely because women value dyadic relationships, they are especially vulnerable in this regard and may experience more intimate loneliness than men. Opposing hypotheses can also be proposed regarding relational loneliness. On the one hand, men might experience less relational loneliness because they orient more toward the group. On the other hand, men may be more vulnerable to relational loneliness because groups are more important for them (Maes, Vanhalst, Van den Noortgate, \& Goossens, 2017). Collective loneliness has received far less attention in the literature and no hypotheses on gender differences in this type of loneliness have been advanced as of yet.

Relationship-specific types of loneliness. Gender differences may vary according to the specific relationship (i.e., with peers, family, or a romantic partner) in which loneliness is experienced. Women might experience less loneliness in relation to the family because they tend to live in a more protected family environment with greater family support than men (Musetti, Corsano, Majorano, \& Mancini, 2012). However, it could also be argued that because the family context is more important for women, they have higher expectations that are more difficult to meet, making them more vulnerable for loneliness (Maes, Klimstra, Van den Noortgate, \& Goossens, 2015). Opposing hypotheses can also be proposed for gender differences in loneliness regarding relationships with peers or a romantic partner (Kuttler \& 


\section{GENDER DIFFERENCES IN LONELINESS}

La Greca, 2004; Maes et al., 2015; Musetti et al., 2012; Zimmer-Gembeck, 2002). It could be argued that women invest more in, and expect more from, their peers and a romantic partner than men. Those higher expectations might not be met, and women might experience more loneliness in those relationships than men. However, it could also be argued that this higher investment leads to higher perceived support, which would result in lower levels of loneliness. Only a few studies examined gender differences in relationship-specific types of loneliness, with results pointing in different directions (e.g., Corsano, Majorano, \& Champretavy, 2006; DiTommaso \& Spinner, 1993; Maes et al., 2015; Musetti et al., 2012; Qualter, Quinton, Wagner, \& Brown, 2009).

Gender differences in loneliness may also depend on the following factors, which were examined in a more exploratory way.

Individualism. Previous studies have found larger gender differences in more individualistic cultures than in more collectivistic cultures regarding personality traits (Costa, Terracciano, \& McCrae, 2001) and the intensity of powerless emotions (i.e., fear, sadness, shame, and guilt; Fischer, Rodriguez Mosquera, van Vianen, \& Manstead, 2004). Given those findings, it is possible that culture is also important for understanding gender differences in loneliness.

Socioeconomic status. In addition, gender differences in loneliness may vary according to the socioeconomic background of the participants. Differences in socialization between males and females may lead to gender differences in values and goals, including occupational choices. For example, males are more likely to want jobs that pay very well and focus more on economic success than females (Eccles, 2007; Pinquart \& Sörensen, 2000). Failure in such economic success, could in turn more strongly influence the well-being of males than females, including feelings of loneliness. Based on these findings, socioeconomic status could also have an effect on gender differences in loneliness. 


\section{GENDER DIFFERENCES IN LONELINESS}

Ethnic majority/minority status. Research on loneliness in ethnic majority and minority groups has been largely inconsistent (Qualter, Maes, \& Nowland, 2016), and research examining whether gender differences in loneliness vary by ethnicity is rare. Metaanalyses on gender differences in related constructs have also yielded inconsistent results, with no influence of ethnicity on gender differences in depressive symptoms (Salk, Hyde, \& Abramson, 2017) and larger gender differences in self-esteem in samples of participants from an ethnic majority than minority group (Kling, Hyde, Showers, \& Buswell, 1999). Exploring whether ethnic majority/minority status acts as a moderator of the relationship between gender and loneliness is important.

Clinical groups. Further, gender differences in loneliness might be dissimilar in clinical and non-clinical samples. Previous meta-analyses have suggested that males and females are differently affected by clinical conditions, such as chronic illnesses. It has, for example, been found that females were more affected by having a chronic condition with depressive symptoms than males (Pinquart \& Shen, 2011b), whereas males tended to react more with externalizing symptoms (Pinquart \& Shen, 2011a). However, when looking at the effects of having a chronic condition on social competence, no gender differences were found (Martinez, Carter, \& Legato, 2011). It is not yet clear whether gender differences in loneliness vary according to clinical status.

Sampling area. Gender differences in loneliness may vary according to the geographical representation of the sample. Studies that sampled participants from just a single site or city might yield less representative results (leading to random error) than studies that sampled from multiple sites or cities in one area, or from multiple geographical areas (such as states or provinces).

Trends over time. Some studies have suggested that the prevalence of mental health problems hasincreased over the years, and that this increase has been stronger for females 


\section{GENDER DIFFERENCES IN LONELINESS}

than males (Bor, Dean, Najman, \& Hayatbakhsh, 2014; Calling, Midlov, Johansson, Sundquist, \& Sundquist, 2017; Sweeting, West, Young, \& Der, 2010). Hence, one could expect larger gender differences in loneliness across time as well.

\section{The Present Study}

Gender differences in loneliness have been frequently examined, but theoretical contentions are conflicting, and findings largely inconsistent (Weeks \& Asher, 2012). Therefore, we aimed to synthesize the available evidence on gender differences in loneliness across the lifespan. In addition to examining that global effect, we were interested in each of the moderator effects as detailed above. Hence, we investigated the effects of participants' age, types of loneliness, country-level individualism, the socioeconomic, ethnic, and clinical status of the participants, the geographical representation of the sample, and trends over time on gender differences in loneliness.

\section{Method}

Because the present study is a meta-analysis of existing publicly available data, our study is exempted by our Institutional Research Board (IRB) from approval.

\section{Literature Search}

Because it is often not clear from the title or abstract of an article whether or not gender differences in loneliness were examined, we aimed to screen the full-text of all empirical reports that included one of the main standardized loneliness measures. Only standardized loneliness questionnaires were included to minimize bias in outcome assessment. These loneliness measures were the Children's Loneliness Scale (CLS; Asher, Hymel, \& Renshaw, 1984), the Differential Loneliness Scale (DLS; Schmidt \& Sermat, 1983), the Loneliness and Aloneness Scale for Children and Adolescents (LACA; Marcoen, Goossens, \& Caes, 1987), the Peer Network and Dyadic Loneliness Scale (PNDLS; Hoza et al., 2000), the Relational Provisions Loneliness Questionnaire (RPLQ; Hayden, 1989), the Rasch-Type 


\section{GENDER DIFFERENCES IN LONELINESS}

Loneliness Scale (RTLS; De Jong Gierveld \& Kamphuis, 1985), the Social and Emotional Loneliness Scale for Adults (SELSA; DiTommaso \& Spinner, 1993), and the University of California Los Angeles Loneliness Scale (UCLA Loneliness Scale; Russell, Peplau, \& Cutrona, 1980). We conducted the literature search in the following databases: PsychInfo, ERIC, PubMed, and Web of Science, using key terms that reflected the names of the loneliness measures. For example, for the UCLA loneliness Scale, we used the search strings ("UCLA Loneliness Scale" or "UCLA Loneliness Questionnaire") and ((UCLA) and (lonel* or "perceived social isola*")). A full list of key terms can be found at the Open Science Framework (https://osf.io/tzg32/). Only empirical journal reports, books, and book chapters were included. This search resulted in 3,594 reports. In addition, we located studies through reports that were obtained in this search and by contacting experts in the field for relevant reports. In this way, we obtained an additional 64 reports. This literature search was completed in August 2014, and the resulting database was labeled as Meta-Analytic Study of Loneliness (MASLO).

\section{Selection of Studies}

The resulting 3,658 reports in the MASLO database were screened (for a flow diagram of the selection process, see Figure 1). Of those reports, 1,376 reports were dropped from the database because they did not use one of the standardized loneliness measures, but only referred to it, for example, in the Introduction. In addition, 206 reports were excluded because they were written in a language other than Dutch, English, French, or German. Finally, we could not retrieve the full-text version of 3 reports. The remaining 2,073 reports were read in depth, after which 248 reports were excluded. Excluded reports included methodologies where a loneliness measure had been administered, but no numeric information for the measure, such as descriptives or univariate statistical tests, was provided. 


\section{GENDER DIFFERENCES IN LONELINESS}

Of the remaining 1,825 reports, 526 reports described gender differences in loneliness in 582 studies (i.e., some reports included multiple studies on multiple datasets). Only unadjusted effect sizes were included (e.g., gender effects examined in multiple regression analyses were not included). Some of the included studies yielded information on multiple effects because they included multiple questionnaires or multiple subscales within a multidimensional questionnaire, resulting in a total of 682 effects. When gender differences were assessed at multiple time points within the same longitudinal study, data were taken from the first measurement wave.

In June 2016, we conducted an update of our procedure, yielding data on an additional 149 effects from 117 reports. Next, we scrutinized all references for duplicates because the same sample of participants is sometimes used in multiple studies. We found 56 such reports and dropped them from the database. We selected the reports that had the most complete data (to calculate effects sizes or code for moderators) and/or the largest sample size. When the available information was equally complete and the samples were equally large, we included

the report that had been published first. The resulting 587 reports yielded 766 effects. For 540 of those effects, sufficient statistical information was reported to calculate an effect size and corresponding standard error. For the other 226 effects, information on gender differences was provided, but insufficient statistics were reported to calculate an effect size and/or the corresponding standard error. For 207 of those 226 effects, we still could calculate an effect size when making assumptions that we will describe in more detail below (see Effect Size Calculations). The final dataset included 747 effect sizes from 634 studies in 571 reports.

\section{Study Coding}

A manual was developed to guide the coding of studies. Undergraduate and graduate students in psychology were trained by the first author to code the reports until they reached a sufficient level of expertise. All reports coded by the students were checked by the first author 


\section{GENDER DIFFERENCES IN LONELINESS}

to verify that the rules described in the manual had been applied correctly. The present dataset included 747 effect sizes $(k)$ from 634 studies $(n)$ in 571 reports published between 1978 and 2016. Sample sizes varied from 10 to 26,116 participants. A total of 398,338 individuals were included in the present meta-analysis, $45.61 \%$ of whom were men. Participants' mean age, as reported in 491 studies, ranged from 5 to 90 years (Mean $=27.80$ years) with a standard deviation, as reported in 374 studies, of 3.99 years.

Age. To examine whether gender differences in loneliness change across developmental periods, we coded for age group. If the age range for a sample spanned more than one category, we chose the category corresponding to the mean age. Studies $(n=634)$ were coded according to the following five age categories: (1) children, that is, participants who were, on average, younger than 12 years or who were in Grade 6 or lower $(n=108)$; (2) adolescents, that is, participants who were between 12 and 21 years old $(n=267)$; (3) young adults, that is, participants who were older than 21 years, but younger than 40 years $(n=96)$; (4) middle age adults, that is, participants who were between 40 and 65 years old $(n=44)$; and (5) elderly, that is, participants who were 65 years or older $(n=70)$.

Loneliness type. To examine whether gender differences vary according to loneliness type, we coded whether the loneliness measures used in the studies reflect (1) intimate, (2) relational, or (3) collective loneliness. Detailed information about the coding of each loneliness measure can be found in the Supplementary Materials. Not all measures could be coded, because some measures, such as the popular UCLA Loneliness Scale, tap into both intimate and relational loneliness. In all, we could code 331 effect sizes $(k, 44.31 \%)$, reflecting intimate loneliness $(k=65)$, relational loneliness $(k=266)$, and collective loneliness $(k=2)$. Because only two effect sizes were available for collective loneliness, we did not include this type of loneliness in the moderator analyses. 


\section{GENDER DIFFERENCES IN LONELINESS}

Relationship-specific types of loneliness. To examine whether gender differences vary according to the specific relationship in which loneliness is experienced, we coded whether the included loneliness scales reflected relationships with (1) peers (including friends and the larger peer group), (2) family (including individual family members and the family as a whole), or (3) a romantic partner (For more details, see Supplementary Materials). Not all measures could be coded because some scales did not refer to a specific relationship. In all, we could code 309 effect sizes $(41.37 \%)$, reflecting loneliness in relationships with peers $(k=$ 236), family $(k=48)$, and a romantic partner $(k=25)$.

Individualism. The studies included in the meta-analysis were conducted in countries from different continents. Most studies (48.90\%) sampled from North America, including the USA $(n=252)$ and Canada $(n=55)$. Studies conducted in Europe comprised $23.34 \%$ of the present dataset, including the Netherlands $(n=31)$, the UK $(n=17)$, Belgium $(n=17)$, and Germany $(n=17)$. Studies conducted in Asia comprised $21.14 \%$ of the present dataset, including China $(n=40)$, Turkey $(n=32)$, and Israel $(n=27)$. Fewer studies were conducted in Oceania (4.73\%), including Australia $(n=29)$ and New Zealand $(n=1)$, and in Africa $(0.63 \%)$, including Zimbabwe $(n=2)$, Nigeria $(n=1)$, and South Africa $(n=1)$. A small subset of studies (1.26\%) could not be categorized, because they used mixed samples from different continents. Some of those studies included participants from South-America, but none of the studies focused solely on participants from that continent.

For each of the 45 countries represented in the present dataset, we took the individualism score from Hofstede's (2001) model of national culture, a score that ranges from 0 to 100. For two of the countries, that is, Zimbabwe and Cuba, no such scores were available, so studies conducted in these countries were not included in the moderator analysis. In all, we could code individualism scores for 617 studies (97.32\%). Individualism scores in the present dataset ranged from 14 to $91(M=72.44, S D=24.38)$. 


\section{GENDER DIFFERENCES IN LONELINESS}

Socioeconomic status. Information regarding the socioeconomic status (SES) of the participants was coded. For many studies, this information was not provided ( $n=378$; $59.62 \%$ ). The other studies comprised (1) low SES samples, with $75 \%$ or more of the participants being of low SES ( $n=36)$; (2) middle or high SES samples, with $75 \%$ or more of the participants being of middle or high SES $(n=81)$; and (3) mixed SES samples, with neither low nor middle or high SES categories making up more than $75 \%$ of the sample $(n=$ 139).

Ethnic majority/minority status. Information regarding the ethnic majority or minority status of the participants was coded. For many studies, this information was not provided ( $n=353 ; 55.68 \%$ ). The other studies were classified as follows: (1) more than $75 \%$ of the participants came from an ethnic minority group $(n=28)$; (2) more than $75 \%$ of the participants came from an ethnic majority group $(n=160)$; and (3) the sample was of mixed ethnic majority/minority status with neither of the categories including more than $75 \%$ ( $n=$ 93).

Clinical groups. To examine whether gender differences varied according to clinical status, we coded whether studies included participants with a physical disability or illness, special educational needs, or mental health problems. We categorized all studies as follows: (1) non-clinical ( $n=556)$, (2) clinical ( $n=51)$, or (3) mixed, that is, studies that looked at both non-clinical and clinical groups $(n=27)$.

Sampling area. To examine whether gender differences in loneliness varied according to the geographical representation of the sample, we coded the studies as follows: (1) participants were sampled in a single city $(n=347)$, (2) participants were sampled in multiple cities within one geographical area $(n=120)$, and (3) participants were sampled in multiple geographical areas $(n=104)$. The remaining 63 studies $(9.94 \%)$ could not be coded due to missing information. 


\section{GENDER DIFFERENCES IN LONELINESS}

Trends over time. To examine trends over time in gender differences, we used year of publication as a proxy. To facilitate interpretation of the estimates, we recoded the values in such a way that the report with the oldest year of publication (i.e., 1978) represented zero.

\section{Effect Size Calculations}

As effect size, we used Hedges' $g$, which is similar to Cohen's $d$ (Rosenthal \& DiMatteo, 2001). We calculated $g$ by subtracting the loneliness mean of women from that of men and dividing the resulting scores by the pooled standard deviation (Lipsey \& Wilson, 2001). A positive effect size, therefore, reflects a higher loneliness mean for men than women. For all effect sizes, we applied Hedges' small-sample correction (Lipsey \& Wilson, 2001). The effect sizes were weighted by the inverse variance (Lipsey \& Wilson, 2001), such that samples with higher precision got a greater weight in the analyses. We interpreted effect sizes based on Cohen's (1988) benchmarks, as suggested by Hyde (2005). So, we interpreted effect sizes as follows: close-to-zero $(g \leq 0.10)$, small $(0.11 \leq g \leq 0.35)$, moderate $(0.36 \leq g \leq$ $0.65)$, large $(0.66 \leq g \leq 1.00)$, or very large $(g>1.00)$.

When descriptive statistics (i.e., means and standard deviations) and sample sizes were provided for men and women separately, we used that information to calculate $g$ and the corresponding standard error. When studies did not provide that information, but provided inferential statistics such as an $F, t$, or $r$ value, we used the formulae presented in Lipsey and Wilson (2001) to derive $g$ and its standard error. Using these conversions, we were able to calculate 540 effect sizes, assuming a common population standard deviation. By making additional assumptions, we were able to calculate an extra 207 effect sizes. For example, if only a total sample size was reported, we assumed an equal sample size for men and women; if the authors reported that no significant gender differences were found, without reporting exact information about the effect size or $p$-value, we assumed an effect size of zero. To 


\section{GENDER DIFFERENCES IN LONELINESS}

assess the sensitivity of our conclusions for the assumptions we made, we performed the meta-analyses with and without the effect sizes for which we had to make assumptions.

\section{Statistical Analyses}

Because several reports reported on multiple studies and multiple effect sizes, we conducted a multilevel meta-analysis. A multilevel meta-analysis does not make the strong assumption of independence that underlies traditional meta-analytic approaches, but explicitly accounts for possible dependencies among effect sizes (Hox, 2002; Van den Noortgate, López-López, Marín-Martínez, \& Sánchez-Meca, 2013). Specifically, we specified a threelevel model. At the first level, there is random sampling variance (which can be very well approximated using the formula presented in Lipsey \& Wilson, 2001, and therefore is assumed to be known in the meta-analysis). At the second level, there is within-study variance, reflecting systematic variance between effect sizes within the same study. At the third level, we considered two sources of random variation, that is, between-study variance (reflecting systematic variance between studies) and between-instrument variance (reflecting systematic variance between the different instruments, that is, scales or - if available subscales, that have been used to assess loneliness). Because the random effects of studies and instruments are not nested but rather crossed (in one study, multiple instruments can be used, and vice versa, the same instrument may be used in multiple studies), this is a cross-classified three-level model (Goldstein, 2003).

To examine whether gender differences varied according to study and sample characteristics, we conducted moderation analyses by including the characteristics as predictors in the three-level cross-classified model. Analyses were conducted with the Metafor package (Version 1.9-9) in R using restricted maximum likelihood (REML) as estimation method (Assink \& Wibbelink, 2016; Viechtbauer, 2010). Overall mean and category-specific mean effects were statistically tested by means of a Wald test, comparing 


\section{GENDER DIFFERENCES IN LONELINESS}

the ratio of the estimate over the corresponding standard error estimate to a $t$-distribution. Moderator effects were tested using Type III $F$-tests. Variance components were tested using a likelihood ratio test, comparing the difference in deviance score of a model including all variance components with a restricted model to a $\mathrm{Chi}^{2}$-distribution. At the Open Science Framework, both the dataset (https://osf.io/h7pj5/) and analysis scripts (https://osf.io/79xsq/) are available.

\section{Results}

\section{Gender Differences in Loneliness}

The 747 observed effect sizes are presented in Figure 2, ordered as a function of increasing support for greater loneliness in men. The $95 \%$ confidence intervals that indicate the precision of each study are also included. This figure, commonly referred to as a caterpillar plot, graphically illustrates that most effect sizes were close to zero. To combine the effect sizes, we conducted three analyses. First, when focusing only on the 540 effects for which sufficient information was available to calculate a standardized mean difference, we found a close-to-zero mean effect of $g=0.08(S E=0.03,95 \%$ CI $[0.02,0.13])$. Although the size of the overall effect is small, it is statistically significant at the .05 significance level, $p=$ .016 , suggesting that men are slightly lonelier than women. Second, when the analysis was based on all 747 effects and, thus, also included the effects for which we had to make assumptions (see 'Effect Size Calculations' above), we obtained a similar effect of $g=0.07$ $(S E=0.02, p=.003$, and 95\% CI [0.03, 0.12]). Third, as an analysis that is more robust against publication bias, we focused on the effect sizes derived from the larger samples with a minimum of 100 male and 100 female participants. This analysis, based on 375 effects, yielded a non-significant mean effect size of $g=0.04$ ( $S E=0.02, p=.078$ and 95\% CI [-0.00, 0.09]). 


\section{GENDER DIFFERENCES IN LONELINESS}

We also examined how the total variance in observed effect sizes was decomposed into sampling variance, within-study, between-study, and between-instrument variance. Because there is no single value for the sampling variance (the variance depends on the size of the study), we used the median sampling variance for this calculation. The within-study variance $\left(0.014, \chi^{2}(1)=87.04, p<.001\right)$ represented $27.57 \%$ of the total variance. The between-study variance $\left(0.012, \chi^{2}(1)=15.794, p<.001\right)$ and the between-instrument variance $\left(0.008, \chi^{2}(1)=17.17, p<.001\right)$ represented $23.44 \%$ and $15.42 \%$ of the total variance, respectively. This means that on top of the sampling variance, there is systematic variance between effect sizes within studies, between studies, and between measurement instruments.

\section{Moderation of Gender Differences in Loneliness}

Most moderators (Table 1) did not significantly predict gender differences in loneliness. Moderators that were not significant were the type of loneliness that was experienced, the relationship in which loneliness was experienced, and the socioeconomic, ethnic minority/majority, and clinical status of the sample. Three of the moderators were significant, that is, age group, sampling area, and year of publication.

First, age group significantly moderated gender differences in loneliness. We found non-significant mean effect sizes for middle age adults and elderly, and small but significant mean effect sizes for children, adolescents, and young adults, suggesting that men were somewhat lonelier than women in those groups. Second, sampling area was found to be a significant moderator, with the largest mean effect size for studies that sampled from a single city. When participants were sampled from multiple cities within a single area or across different geographical areas, the mean estimated effect sizes were no longer significant. Third, gender differences in loneliness tended to become smaller in more recently published reports. These three moderators together explained $12.72 \%$ of the systematic variance.

\section{Publication Bias}




\section{GENDER DIFFERENCES IN LONELINESS}

The mean effect size slightly decreased when we excluded studies with small sample sizes, suggesting that there might be publication bias as well. Therefore, we examined the presence of publication bias in two additional ways. First, to obtain a rough indication of publication bias, we created a funnel plot (Figure 3) for those studies with sufficient statistical information to compute an effect size $(k=540)$ and for the total dataset including the studies for which we had to make additional assumptions $(k=747)$. In the absence of publication bias, we would expect these plots to be shaped as a funnel, suggesting that as sample size increases, studies converge more closely around the true mean. The two plots, which were highly similar, effectively showed more or less a funnel shape. To test statistically for publication bias, we applied an extension of Egger's regression test by adding the sampling variance as a moderator to the model $(k=747)$. That moderator did not reach significance, $F(1,745)=0.85, p=.357$. Hence, publication bias was unlikely to have had a substantial influence on our findings.

\section{Discussion}

The present meta-analysis examined gender differences in loneliness across the lifespan. Overall, we found a small but significant effect suggesting that males are slightly lonelier than females. When focusing on only those samples with at least 100 males and 100 females, the effect was not significant. Gender differences varied according to the age of the participants, the scope of the sampling area, and the year of publication. No significant effects were found for the other moderators, that is, types of loneliness, country-level individualism, and the socioeconomic, ethnic, and clinical status of the participants.

\section{Moderating Factors}

Given that previous theoretical and empirical work suggested gender differences might be limited to certain age groups only (Martel, 2013; Pinquart \& Sörensen, 2001), we examined gender differences in loneliness separately for different age groups. We found that 


\section{GENDER DIFFERENCES IN LONELINESS}

for children, adolescents, and young adults, males were slightly lonelier than females; for middle age adults and elderly no significant gender effects were found. As indicated in the Introduction, theoretical models of internalizing problems (e.g., Martel, 2013) predict that women are lonelier than men from adolescence onwards. Our results suggest that the age trend actually goes in the opposite direction (i.e., decreasing gender differences with advancing age) and that overall women are no more vulnerable to loneliness than men.

The largest gender differences were found for young adults (i.e., aged between 21 and 40 years). A recent study, based on a large nationally representative German sample of adults, found a peak in loneliness for this age group (Luhmann \& Hawkley, 2016). That study also examined which predictors of loneliness were specific for which age group. For young adults, they found three age-specific predictors of loneliness, that is, income, having a full-time job, and relationship status. According to the age-normative perspective, individuals are less lonely when they meet their age-normative expectations. Young adulthood is the period in life when making and saving money, building a career, and finding a partner and starting a family are more important life goals than in other periods in life (Luhmann \& Hawkley, 2016). Moreover, previous research showed that economic success was more important for males than females (Eccles, 2007; Pinquart \& Sörensen, 2000). So, not meeting the norm of making money and building a career might lead to loneliness among young adults, and these effects might be stronger for males. Regarding the third predictor, that is, relationship status, it is not yet clear whether this would be a more important life goal for males or females in this period of life.

Moreover, we should be rather cautious with our conclusions because the gender difference found for young adults was significant, but also rather small $(g=0.12)$. This effect is in line with previous meta-analyses on gender differences in several psychological variables, which consistently found rather small effects (with some exceptions, including 


\section{GENDER DIFFERENCES IN LONELINESS}

some motor behaviors and some aspects of sexuality; Hyde, 2005). An effect of $g=0.12$ means that average loneliness scores of men and women differ from each other by 0.12 of a standard deviation. Phrased in another way, our results show for young adults that $24 \%$ of males is at least as lonely as the $20 \%$ most lonely women.

In addition to age category, we examined whether gender differences in loneliness would vary according to the different types of loneliness. Gender differences did not vary according to the type of loneliness examined (i.e., intimate or relational loneliness), nor the relationship context in which loneliness was experienced (i.e., peers, family, or a romantic partner). This finding is in line with previous research, showing that even though males and females tend to focus on and value different types of relationships, they are equally social and take comparable care in how they relate to others (Baumeister \& Sommer, 1997; Gardner \& Gabriel, 2004; Rose \& Rudolph, 2006).

Regarding the other moderators tested, gender differences in loneliness did not vary according to the degree of individualism of the country from which the participants were sampled, or the socioeconomic, ethnic minority/majority, and clinical status of the participants. Loneliness is a universal phenomenon, and these results suggest that males and females are very similar regarding mean levels of loneliness, across a wide range of different contexts.

Gender differences did vary according to the scope of the sampling area and year of publication. We found the largest gender differences in loneliness for studies that sampled from a single city. However, gender differences disappeared for studies that sampled from multiple cities within a single geographical area and across multiple geographical areas. As hypothesized, it might be that studies that sampled participants from just a single city yield less representative results, leading to random error, than studies that sampled from multiple cities and/or geographical areas. Furthermore, a small effect of publication year was found, 


\section{GENDER DIFFERENCES IN LONELINESS}

suggesting that more recent studies show smaller gender differences in loneliness, which might represent a greater willingness to discuss loneliness in both sexes and a closing of the gender gap in relation to mental health.

\section{Recommendations for Future Research}

Reviewing the literature on gender differences in loneliness across the lifespan led us to several suggestions for future research. As a research community, we should aim to base our conclusions on a set of studies that is representative and covers the human population as well as possible. For example, over $75 \%$ of the studies in the present meta-analysis were conducted in Western countries, with about half of these from the US. Although research outside the US is increasing, information for some parts of the world, especially Africa and South-America, is largely lacking. Also, more than half of all studies included in the present meta-analysis did not report information about the socioeconomic or ethnic status of the participants. We would like to urge researchers to include information on those demographic characteristics of their sample in their research reports. Of those studies that reported such information, only $14 \%$ included samples with mostly participants of low SES and only $10 \%$ included samples with mostly participants from an ethnic minority group. Furthermore, 26\% of all studies focused on college students, which represents a very specific context that is only experienced by a limited number of people, typically people with higher SES and belonging to the majority group (Henrich, Heine, \& Norenzayan, 2010). Overall, we strongly suggest future research to report on demographics of the sample, and to include populations that are less frequently studied and more difficult to reach, in order to expand our knowledge base and to generalize our findings.

Consideration also needs to be given to providing sufficient statistical information regarding gender differences in future studies. When studies found a non-significant gender difference, but reported insufficient statistics to compute a standardized mean difference, we 


\section{GENDER DIFFERENCES IN LONELINESS}

entered a conservative effect size of zero. An effect size is unlikely to be exactly zero, and it might be that all these effect sizes actually were in a certain direction. However, our results on the subset of studies with sufficient information to calculate an effect size yielded a similar, close-to-zero effect. Nevertheless, we encourage researchers to also report sufficient information for non-significant results.

Finally, future research should examine different types of loneliness more systematically. Regarding the third type of loneliness distinguished in the Introduction, that is, collective loneliness, conceptual and empirical work is largely lacking. Distinguishing among different types of loneliness is not only of interest for gender differences, but is important in its own right. For example, previous research on adolescents found that different types of loneliness were related to problems in different domains (e.g., parenting and peer group functioning; Maes, Vanhalst, Spithoven, Van den Noortgate, \& Goossens, 2016) and to different forms of psychopathology (Lasgaard, Goossens, Bramsen, Trillingsgaard, \& Elklit, 2011).

\section{Limitations}

First, samples were categorized into five different age groups, representing important developmental periods across life. This categorization was performed based on the mean age of the sample. However, not all samples were age-homogeneous, and some actually contained participants from different age categories. Second, although we searched thoroughly and systematically in various databases, searching additional databases (such as Embase) possibly could have resulted in additional findings. Third, we did not have a second coder for each study. However, several efforts were made to increase coding consistency, that is, we created a detailed coding manual, developed an extensive training for the coders, and checked all reports coded to verify that the rules described in the manual had been applied correctly. Fourth, because studies usually do not specify how they assessed ethnicity, we could not code 


\section{GENDER DIFFERENCES IN LONELINESS}

for it. This means, for example, that we do not know which generations of immigrants were included when authors referred to 'ethnic minorities'. Finally, please note that we focused on gender differences in mean levels of loneliness. It may be that sources and outcomes of loneliness differ for men and women.

\section{Practical Implications}

Overall, we found significant gender differences in loneliness for children, adolescents, and young adults. However, those effects were very small. Thus, researchers, policy makers, and practitioners should not assume men to be more lonely than women and should develop and offer interventions for both. Indeed, when we let our prejudices about gender influence us, it means the group we view as less lonely risks receiving less recognition and treatment by professionals (Borys \& Perlman, 1985; Salk et al., 2017). Our results suggest that mean levels of loneliness across the lifespan are similar for males and females, and advertisements of services and interventions should be directed to both. However, this does not mean that we should assume a "one size fits all" intervention. Different types of loneliness may need different intervention strategies.

\section{Conclusion}

To conclude, our review into gender differences in loneliness covered 39 years of research, including samples with various socioeconomic, ethnic minority/majority, and clinical status from 45 countries. Overall, we did not find strong evidence for gender differences in loneliness, suggesting that males and females are more alike than they are different in this regard. 


\section{GENDER DIFFERENCES IN LONELINESS}

\section{References}

Asher, S. R., Hymel, S., \& Renshaw, P. D. (1984). Loneliness in children. Child Development, 55, 1456-1464. doi:10.2307/1130015

Assink, M., \& Wibbelink, C. J. (2016). Fitting three-level meta-analytic models in R: A stepby-step tutorial. Quantitative Methods for Psychology, 12, 154-174. doi:10.20982/tqmp.12.3.p154

Baumeister, R. F., \& Sommer, K. L. (1997). What do men want? Gender differences and two spheres of belongingness: Comment on Cross and Madson (1997). Psychological Bulletin, 122, 38-44. doi:10.1037/0033-2909.122.1.38

Bor, W., Dean, A. J., Najman, J., \& Hayatbakhsh, R. (2014). Are child and adolescent mental health problems increasing in the 21 st century? A systematic review. Australian and New Zealand Journal of Psychiatry, 48, 606-616. doi:10.1177/0004867414533834

Borys, S., \& Perlman, D. (1985). Gender differences in loneliness. Personality and Social Psychology Bulletin, 11, 63-74. doi:10.1177/0146167285111006

Cacioppo, J. T., Cacioppo, S., Cole, S. W., Capitanio, J. P., Goossens, L., \& Boomsma, D. I. (2015). Loneliness across phylogeny and a call for comparative studies and animal models. Perspectives on Psychological Science, 10, 202-212. doi:10.1177/1745691614564876

Cacioppo, S., Grippo, A. J., London, S., Goossens, L., \& Cacioppo, J. T. (2015). Loneliness: Clinical import and interventions. Perspectives on Psychological Science, 10, 238249. doi:10.1177/1745691615570616

Calling, S., Midlov, P., Johansson, S. E., Sundquist, K., \& Sundquist, J. (2017). Longitudinal trends in self-reported anxiety. Effects of age and birth cohort during 25 years. $B M C$ Psychiatry, 17, 119-129. doi:10.1186/s12888-017-1277

Cohen, J. (1988). Statistical power analysis for the behavioral sciences (2nd ed.). Hillsdale, NJ: Erlbaum.

Corsano, P., Majorano, M., \& Champretavy, L. (2006). Psychological well-being in adolescence: The contribution of interpersonal relations and experience of being alone. Adolescence, 41, 341-353. 


\section{GENDER DIFFERENCES IN LONELINESS}

Costa, P. T., Jr., Terracciano, A., \& McCrae, R. R. (2001). Gender differences in personality traits across cultures: Robust and surprising findings. Journal of Personality and Social Psychology, 81, 322-331. doi:10.1037/0022-3514.81.2.322

Creemers, D. H., Scholte, R. H., Engels, R. C., Prinstein, M. J., \& Wiers, R. W. (2012). Implicit and explicit self-esteem as concurrent predictors of suicidal ideation, depressive symptoms, and loneliness. Journal of Behavior Therapy and Experimental Psychiatry, 43, 638-646. doi:10.1016/j.jbtep.2011.09.006

Csikszentmihalyi, M., \& Larson, R. (1984). Being adolescent: Conflict and growth in the teenage years. New York, NY: Basic Books.

De Jong Gierveld, J., \& Kamphuis, F. (1985). The development of a Rasch-Type loneliness scale. Applied Psychological Measurement, 9, 289-299. doi:10.1177/014662168500900307

DiTommaso, E., \& Spinner, B. (1993). The development and initial validation of the Social and Emotional Loneliness Scale for Adults (SELSA). Personality and Individual Differences, 14, 127-134. doi:10.1016/0191-8869(93)90182-3

Doane, L. D., \& Thurston, E. C. (2014). Associations among sleep, daily experiences, and loneliness in adolescence: Evidence of moderating and bidirectional pathways. Journal of Adolescence, 37, 145-154. doi:10.1016/j.adolescence.2013.11.009

Eccles, J. S. (2007). Families, schools, and developing achievement-related motivations and engagement. In J. E. Grusec \& P. D. Hastings (Eds.), Handbook of socialization: Theory and research (pp. 665-691). New York, NY: Guilford Press.

Ernst, J. M., \& Cacioppo, J. T. (1999). Lonely hearts: Psychological perspectives on loneliness. Applied and Preventive Psychology, 8, 1-22. doi:10.1016/s09621849(99)80008-0

Fischer, A. H., Rodriguez Mosquera, P. M., van Vianen, A. E. M., \& Manstead, A. S. R. (2004). Gender and culture differences in emotion. Emotion, 4, 87-94. doi:10.1037/1528-3542.4.1.87 


\section{GENDER DIFFERENCES IN LONELINESS}

Gardner, W. L., \& Gabriel, S. (2004). Gender differences in relational and collective interdependence: Implications for self-views, social behavior, and subjective wellbeing. In A. H. Eagly, A. E. Beall, \& R. J. Sternberg (Eds.), The psychology of gender (2nd ed., pp. 169-191). New York, NY: Guilford Press.

Goldstein, H. (2003). Multilevel statistical models (3rd ed.). London, UK: Arnold.

Goossens, L., van Roekel, E., Verhagen, M., Cacioppo, J. T., Cacioppo, S., Maes, M., \& Boomsma, D. I. (2015). The genetics of loneliness: Linking evolutionary theory to genome-wide genetics, epigenetics, and social science. Perspectives on Psychological Science, 10, 213-226. doi:10.1177/1745691614564878

Harris, R. A., Qualter, P., \& Robinson, S. J. (2013). Loneliness trajectories from middle childhood to pre-adolescence: Impact on perceived health and sleep disturbance. Journal of Adolescence, 36, 1295-1304. doi:10.1016/j.adolescence.2012.12.009

Hawkley, L. C., \& Capitanio, J. P. (2015). Perceived social isolation, evolutionary fitness and health outcomes: A lifespan approach. Philosophical Transactions of the Royal Society B, 370, 20140114. doi:10.1098/rstb.2014.0114

Hayden, L. K. (1989). Children's loneliness. Unpublished doctoral dissertation. University of Waterloo. Waterloo, Ontario, Canada.

Heinrich, L. A., \& Gullone, E. (2006). The clinical significance of loneliness: A literature review. Clinical Psychology Review, 26, 695-718. doi:10.1016/j.cpr.2006.04.002

Henrich, J., Heine, S. J., \& Norenzayan, A. (2010). Most people are not WEIRD. Nature, 466, 29-29. doi:10.1038/466029a

Hofstede, G. (2001). Culture's consequences: Comparing values, behaviors, institutions, and organizations across nations (2nd ed.). Thousand Oaks, CA: Sage.

Holt-Lunstad, J., Smith, T. B., Baker, M., Harris, T., \& Stephenson, D. (2015). Loneliness and social isolation as risk factors for mortality: A meta-analytic review. Perspectives on Psychological Science, 10, 227-237. doi:10.1177/1745691614568352 


\section{GENDER DIFFERENCES IN LONELINESS}

Hox, J. (2002). Multilevel analysis: Techniques and applications. Mahwah, NJ: Erlbaum.

Hoza, B., Bukowski, W. M., \& Beery, S. (2000). Assessing peer network and dyadic loneliness. Journal of Clinical Child Psychology, 29, 119-128. doi:10.1207/S15374424jccp2901_12

Hyde, J. S. (2005). The gender similarities hypothesis. American Psychologist, 60, 581-592. doi:10.1037/0003-066X.60.6.581

Kling, K. C., Hyde, J. S., Showers, C. J., \& Buswell, B. N. (1999). Gender differences in selfesteem: A meta-analysis. Psychological Bulletin, 125, 470-500. doi:10.1037/00332909.125.4.470

Koenig, L. J., \& Abrams, R. F. (1999). Adolescent loneliness and adjustment: A focus on gender differences. In K. J. Rotenberg \& S. Hymel (Eds.), Loneliness in childhood and adolescence (pp. 296-322). New York, NY: Cambridge University Press.

Kuttler, A. F., \& La Greca, A. M. (2004). Linkages among adolescent girls' romantic relationships, best friendships, and peer networks. Journal of Adolescence, 27, 395414. doi:10.1016/j.adolescence.2004.05.002

Larson, R. W. (1990). The solitary side of life: An examination of the time people spend alone from childhood to old age. Developmental Review, 10, 155-183. doi:10.1016/0273-2297(90)90008-R

Lasgaard, M., Goossens, L., Bramsen, R. H., Trillingsgaard, T., \& Elklit, A. (2011). Different sources of loneliness are associated with different forms of psychopathology in adolescence. Journal of Research in Personality, 45, 233-237. doi:10.1016/j.jrp.2010.12.005

Lipsey, M. W., \& Wilson, D. B. (2001). Practical meta-analysis. Thousand Oaks, CA: Sage. Luhmann, M., \& Hawkley, L. C. (2016). Age differences in loneliness from late adolescence to oldest old age. Developmental Psychology, 52, 943-959. doi:10.1037/dev0000117

Maes, M., Klimstra, T., Van den Noortgate, W., \& Goossens, L. (2015). Factor structure and measurement invariance of a multidimensional loneliness scale: Comparisons across gender and age. Journal of Child and Family Studies, 24, 1829-1837.

doi:10.1007/s 10826-014-9986-4 


\section{GENDER DIFFERENCES IN LONELINESS}

Maes, M., Vanhalst, J., Spithoven, A. M., Van den Noortgate, W., \& Goossens, L. (2016). Loneliness and attitudes toward aloneness in adolescence: A person-centered approach. Journal of Youth and Adolescence, 45, 547-567. doi:10.1007/s10964-0150354-5

Maes, M., Vanhalst, J., Van den Noortgate, W., \& Goossens, L. (2017). Intimate and relational loneliness in adolescence. Journal of Child and Family Studies, 26, 20592069. doi:10.1007/s10826-017-0722-8

Marcoen, A., Goossens, L., \& Caes, P. (1987). Loneliness in pre through late adolescence: Exploring the contributions of a multidimensional approach. Journal of Youth and Adolescence, 16, 561-577. doi:10.1007/bf02138821

Martel, M. M. (2013). Sexual selection and sex differences in the prevalence of childhood externalizing and adolescent internalizing disorders. Psychological Bulletin, 139, 1221-1259. doi:10.1037/a0032247

Martinez, W., Carter, J. S., \& Legato, L. J. (2011). Social competence in children with chronic illness: A meta-analytic review. Journal of Pediatric Psychology, 36, 878-890. doi:10.1093/jpepsy/jsr035

Musetti, A., Corsano, P., Majorano, M., \& Mancini, T. (2012). Identity processes and experience of being alone during late adolescence. International Journal of Psychoanalysis and Education, 1, 44-65.

Perlman, D., \& Peplau, L. A. (1981). Toward a social psychology of loneliness. In S. Duck \& R. Gilmour (Eds.), Personal relationships in disorder (Vol. 3, pp. 31-56). London, UK: Academic Press.

Pinquart, M. \& Shen, Y. (2011a). Behavior problems in children and adolescents with chronic physical illness: A meta-analysis. Journal of Pediatric Psychology, 36, 1003-1016. doi:10.1093/jpepsy/jsr042

Pinquart, M. \& Shen, Y. (2011b). Depressive symptoms in children and adolescents with chronic physical illness: An updated meta-analysis. Journal of Pediatric Psychology, 36, 375-384. doi:10.1093/jpepsy/jsq104 


\section{GENDER DIFFERENCES IN LONELINESS}

Pinquart, M. \& Sörensen, S. (2000). Influences of socioeconomic status, social network, and competence on subjective well-being in later life: A meta-analysis. Psychology and Aging, 15, 187-224. doi:10.1037/0882-7974.15.2.187

Pinquart, M., \& Sörensen, S. (2001). Gender differences in self-concept and psychological well-being in old age: A meta-analysis. Journals of Gerontology: Series B: Psychological Sciences and Social Sciences, 56B, P195-P213. doi:10.1093/geronb/56.4.P195

Qualter, P., Brown, S. L., Rotenberg, K. J., Vanhalst, J., Harris, R. A., Goossens, L., . . Munn, P. (2013). Trajectories of loneliness during childhood and adolescence: Predictors and health outcomes. Journal of Adolescence, 36, 1283-1293. doi:10.1016/j.adolescence.2013.01.005

Qualter, P., Maes, M., \& Nowland, R. (2016). Loneliness. In B. Carducci (Ed.), Encyclopedia of personality and individual differences: Volume II. Chichester, UK: Wiley.

Qualter, P., Quinton, S. J., Wagner, H., \& Brown, S. (2009). Loneliness, interpersonal distrust, and alexithymia in university students. Journal of Applied Social Psychology, 39, 1461-1479. doi:10.1111/j.1559-1816.2009.00491.x

Qualter, P., Vanhalst, J., Harris, R. A., Van Roekel, E., Lodder, G., Bangee, M., . . . Verhagen, M. (2015). Loneliness across the life span. Perspectives on Psychological Science, 10, 250-264. doi:10.1177/1745691615568999

Romero, L. E., \& Epkins, C. C. (2008). Girls' cognitions of hypothetical friends: Are they related to depression, loneliness, social anxiety and perceived similarity? Journal of Social and Personal Relationships, 25, 311-332. doi:10.1177/0265407507087961

Rose, A. J., \& Rudolph, K. D. (2006). A review of sex differences in peer relationship processes: Potential trade-offs for the emotional and behavioral development of girls and boys. Psychological Bulletin, 132, 98-131. doi:10.1037/0033-2909.132.1.98

Rosenthal, R., \& DiMatteo, M. R. (2001). Meta-analysis: Recent developments in quantitative methods for literature reviews. Annual Review of Psychology, 52, 59-82. doi:10.1146/annurev.psych.52.1.59 


\section{GENDER DIFFERENCES IN LONELINESS}

Russell, D., Peplau, L. A., \& Cutrona, C. E. (1980). The Revised UCLA Loneliness Scale: Concurrent and discriminant validity evidence. Journal of Personality and Social Psychology, 39, 472-480. doi:10.1037/0022-3514.39.3.472

Salk, R. H., Hyde, J. S., \& Abramson, L. Y. (2017). Gender differences in depression in representative national samples: Meta-analyses of diagnoses and symptoms. Psychological Bulletin, 143, 783-822. doi:10.1037/bu10000102

Schmidt, N., \& Sermat, V. (1983). Measuring loneliness in different relationships. Journal of Personality and Social Psychology, 44, 1038-1047. doi:10.1037/0022-3514.44.5.1038

Sweeting, H., West, P., Young, R., \& Der, G. (2010). Can we explain increases in young people's psychological distress over time? Social Science and Medicine, 71, 18191830. doi:10.1016/j.socscimed.2010.08.012

Van den Noortgate, W., López-López, J. A., Marín-Martínez, F., \& Sánchez-Meca, J. (2013). Three-level meta-analysis of dependent effect sizes. Behavior Research Methods, 45, 576-594. doi:10.3758/s13428-012-0261-6

Vanhalst, J., Klimstra, T. A., Luyckx, K., Scholte, R. H., Engels, R. C., \& Goossens, L. (2012). The interplay of loneliness and depressive symptoms across adolescence: Exploring the role of personality traits. Journal of Youth and Adolescence, 41, 776787. doi:10.1007/s10964-011-9726-7

Victor, C. R., \& Yang, K. (2012). The prevalence of loneliness among adults: A case study of the United Kingdom. Journal of Psychology: Interdisciplinary and Applied, 146, 85104. doi:10.1080/00223980.2011.613875

Viechtbauer, W. (2010). Conducting meta-analyses in R with the Metafor package. Journal of Statistical Software, 36, 1-48. doi:10.18637/jss.v036.i03

Weeks, M. S., \& Asher, S. R. (2012). Loneliness in childhood: Toward the next generation of assessment and research. Advances in Child Development and Behavior, 42, 1-39. doi:10.1016/B978-0-12-394388-0.00001-0

Zimmer-Gembeck, M. J. (2002). The development of romantic relationships and adaptations in the system of peer relationships. Journal of Adolescent Health, 31, 216-225. doi:10.1016/S1054-139X(02)00504-9 


\section{GENDER DIFFERENCES IN LONELINESS}

Table 1

Separate Regression Analyses for the Moderators Predicting Gender Differences in Loneliness

\begin{tabular}{|c|c|c|c|c|c|c|c|}
\hline Moderator & $k$ & $\beta$ & $S E \beta$ & $95 \% \mathrm{CI}$ & $F$ & $\overline{d f}$ & $\bar{p}$ \\
\hline Age & 690 & & & & 7.51 & 4,685 & $<.001$ \\
\hline Children & 114 & $0.08_{\mathrm{b}, \mathrm{c}}$ & 0.03 & $0.01,0.14$ & & & .029 \\
\hline Adolescents & 307 & $0.08_{b, c}$ & 0.03 & $0.03,0.13$ & & & .002 \\
\hline Young adults & 133 & $0.12_{\mathrm{c}}$ & 0.03 & $0.06,0.18$ & & & $<.001$ \\
\hline Middle age adults & 53 & $0.02_{\mathrm{a}, \mathrm{b}}$ & 0.03 & $-0.05,0.09$ & & & .541 \\
\hline Elderly & 83 & $-0.05_{\mathrm{a}}$ & 0.03 & $-0.11,0.02$ & & & .134 \\
\hline Loneliness type & 331 & & & & 1.60 & 1,329 & .206 \\
\hline Intimate & 65 & 0.04 & 0.06 & $-0.08,0.16$ & & & .546 \\
\hline Relational & 266 & 0.13 & 0.05 & $0.04,0.23$ & & & .006 \\
\hline Relationship & 309 & & & & 0.14 & 2,306 & .867 \\
\hline Peers & 236 & 0.05 & 0.04 & $-0.04,0.13$ & & & .267 \\
\hline Family & 48 & 0.08 & 0.07 & $-0.06,0.21$ & & & .274 \\
\hline Romantic partner & 25 & 0.02 & 0.09 & $-0.17, \quad 0.20$ & & & .871 \\
\hline Individualism & 726 & -0.00 & 0.00 & $-0.00, \quad 0.00$ & 3.12 & 1,724 & .078 \\
\hline Socioeconomic status & 286 & & & & 1.08 & 2,283 & .340 \\
\hline Mostly low SES & 40 & -0.03 & 0.05 & $-0.12,0.06$ & & & .523 \\
\hline Mixed SES & 157 & 0.01 & 0.04 & $-0.06,0.09$ & & & .705 \\
\hline Mostly Middle/high SES & 89 & 0.03 & 0.04 & $-0.05,0.11$ & & & .452 \\
\hline Ethnic majority/minority & 315 & & & & 2.61 & 2,312 & .075 \\
\hline Mostly minority & 29 & 0.02 & 0.05 & $-0.08,0.12$ & & & .697 \\
\hline Mixed minority/majority & 103 & 0.11 & 0.03 & $0.04,0.18$ & & & .001 \\
\hline Mostly majority & 183 & 0.06 & 0.03 & $0.00,0.12$ & & & .035 \\
\hline
\end{tabular}




\section{GENDER DIFFERENCES IN LONELINESS}

\begin{tabular}{|c|c|c|c|c|c|c|c|c|}
\hline Clinical status & 747 & & & & & 1.04 & 2,744 & .354 \\
\hline Non-clinical & 662 & 0.08 & 0.02 & 0.03, & 0.12 & & & .002 \\
\hline Mixed & 27 & 0.04 & 0.05 & -0.06 & 0.14 & & & .409 \\
\hline Clinical & 58 & 0.03 & 0.04 & -0.05 & 0.11 & & & .470 \\
\hline Sampling area & 671 & & & & & 7.43 & 2,668 & $<.001$ \\
\hline Single city & 403 & $0.10_{\mathrm{a}}$ & 0.03 & 0.05 , & 0.15 & & & $<.001$ \\
\hline Multiple cities, single area & 135 & $0.05 \mathrm{~b}$ & 0.03 & -0.00 & 0.11 & & & .060 \\
\hline Multiple areas & 133 & $0.02 \mathrm{~b}$ & 0.03 & -0.03 & 0.07 & & & .471 \\
\hline Publication year & 747 & -0.00 & 0.00 & -0.00 & -0.00 & 7.55 & 1,745 & .006 \\
\hline
\end{tabular}

Note. The regression coefficients for the categorical variables can be interpreted as the mean effect sizes for each category. $k$ is the number of effect sizes in the category; $\beta=$ regression coefficient; CI $=$ confidence interval. Effects sizes are significantly different if they do not have the same subscript. 


\section{GENDER DIFFERENCES IN LONELINESS}

\section{Literature search}

Databases: PsycInfo, ERIC, PubMed, and Web of Science

Checking reference lists

Contacting experts in the field

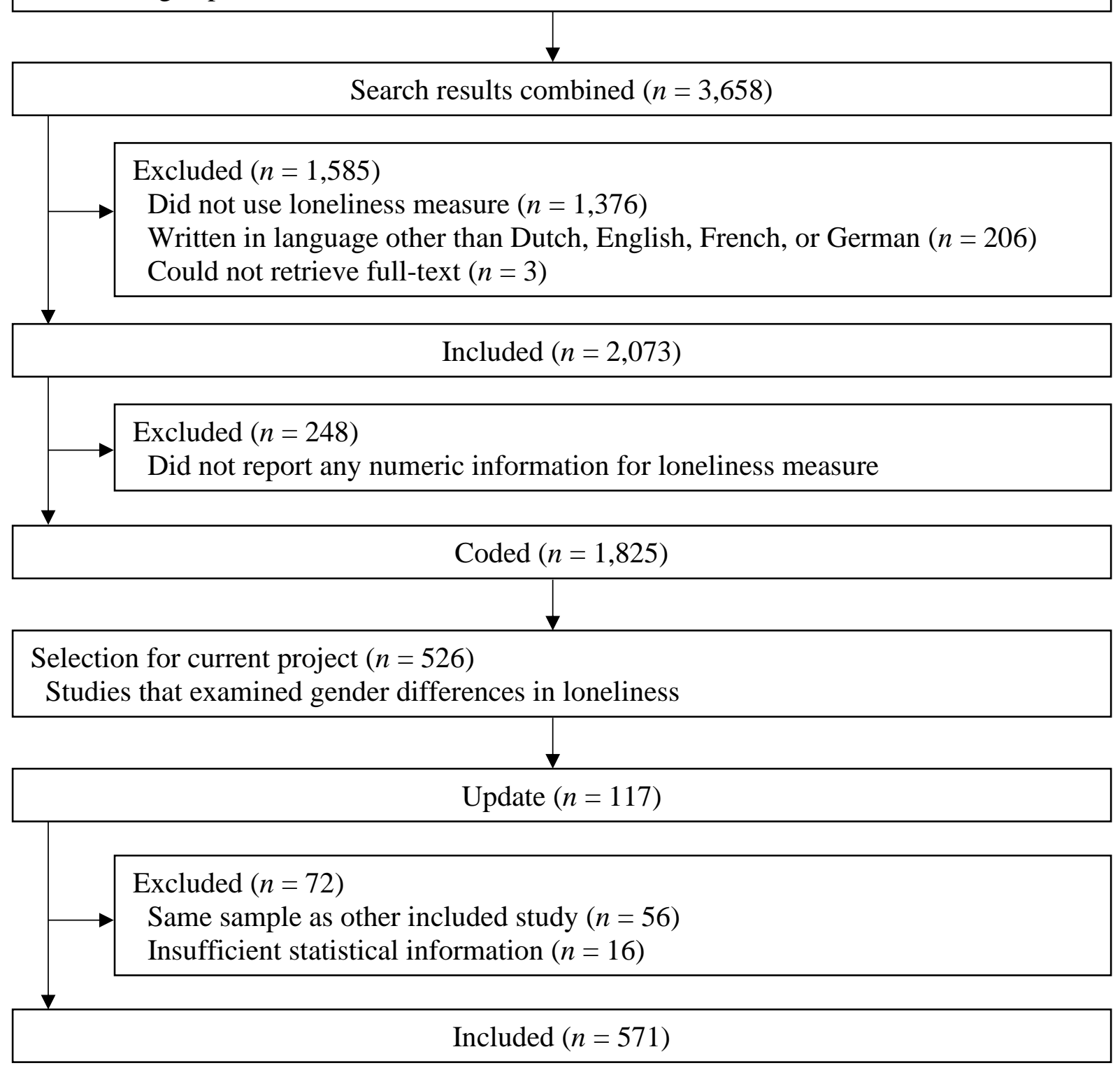

Figure 1. PRISMA flow diagram. 


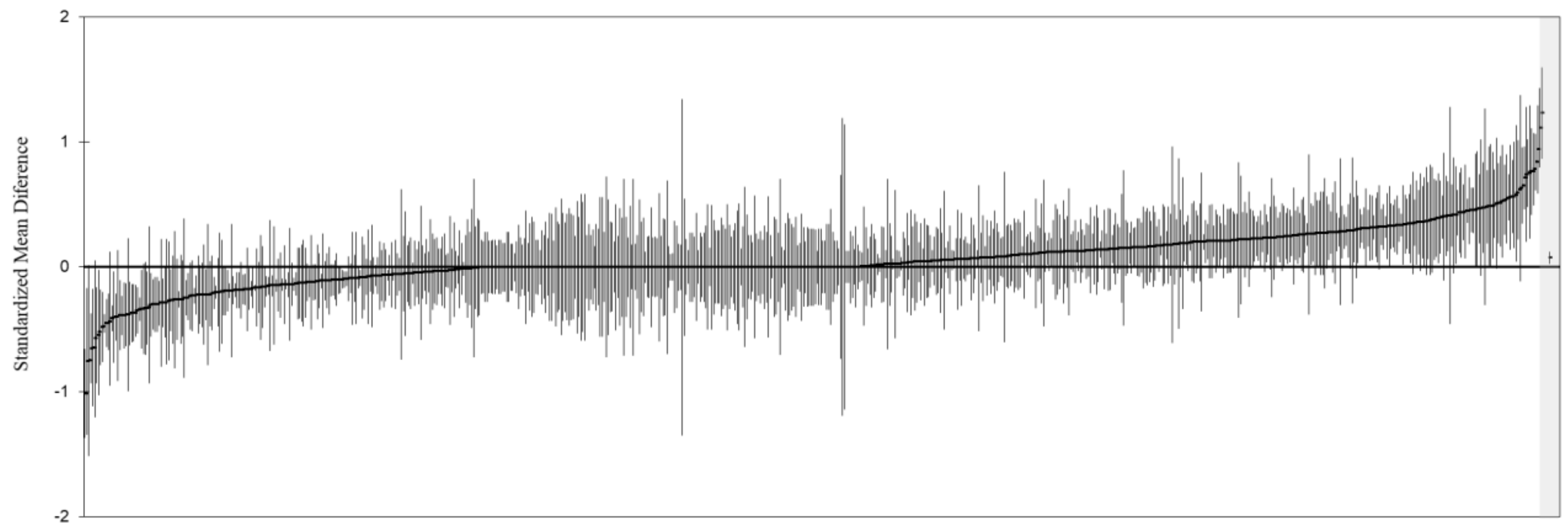

$=$ Mean ES REM

Figure 2. Caterpillar plot of the observed effect sizes for gender differences in loneliness with 95\% confidence intervals. 
A

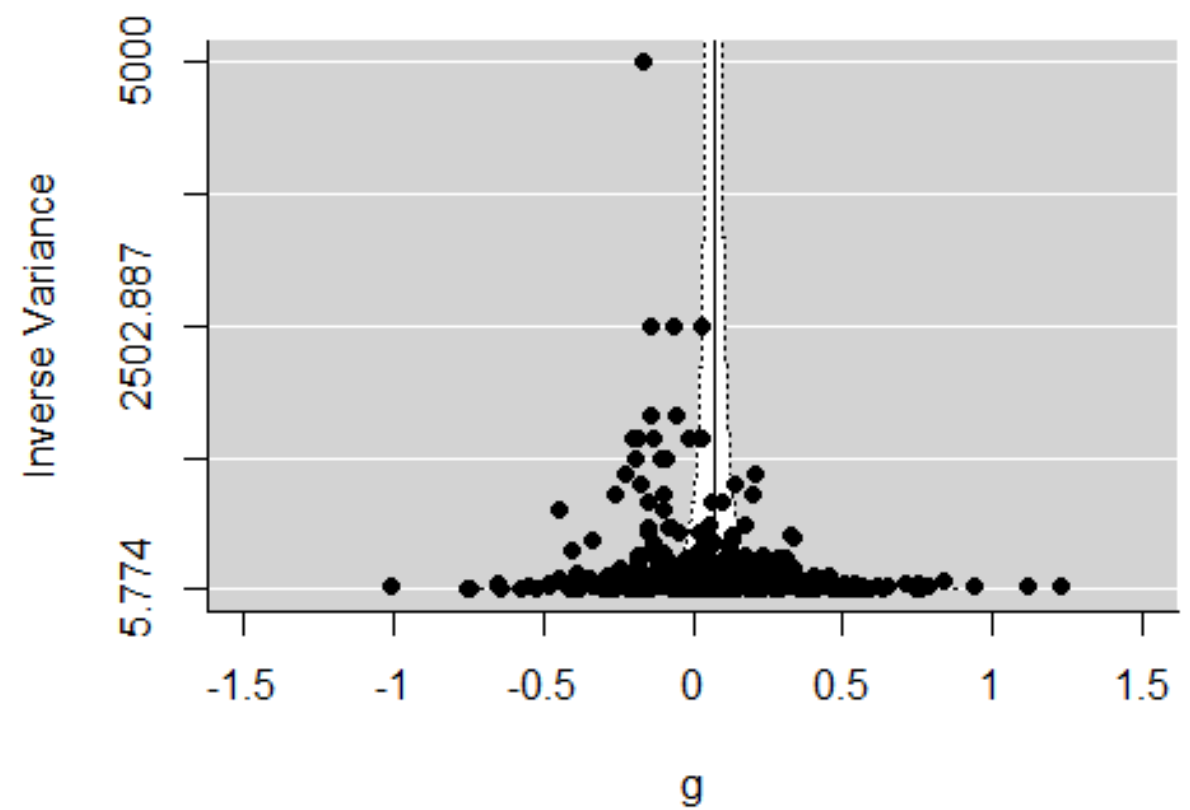

B

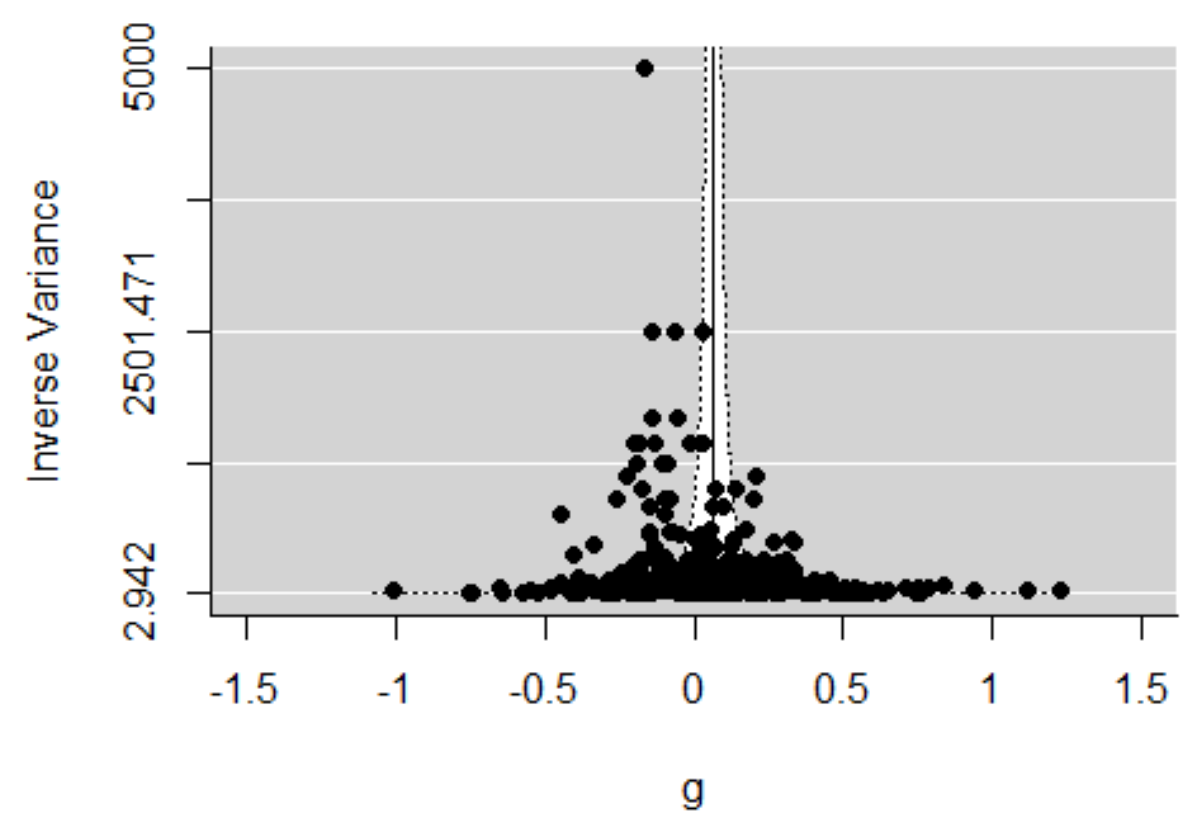

Figure 3. Funnel plots of effect sizes. Effect size $(g)$ is plotted on the $\mathrm{x}$-axis and the number of participants on the y-axis. Panel A represent the 540 effect sizes for which sufficient information was available to calculate $g$. Panel B represents the total dataset of 747 effect sizes. 